\title{
Role of Human Mesangial-Tubular Crosstalk in Secretory IgA-Induced IgA Nephropathy
}

\author{
Junjun Zhang ${ }^{\mathrm{a}}$ Ruwen Zhou ${ }^{\mathrm{a}, \mathrm{b}}$ Yiming Mi ${ }^{\mathrm{a}}$ Zhangsuo Liu ${ }^{\mathrm{a}, \mathrm{b}, \mathrm{c}, \mathrm{d}}$ \\ Bo Huang $^{a}$ Ruxue Guo ${ }^{a, b}$ Panfei Wang ${ }^{a, b}$ Songxia Quan ${ }^{e}$ Yali Zhou ${ }^{e}$ \\ aDepartment of Nephrology, The First Affiliated Hospital of Zhengzhou University, Zhengzhou, China; \\ ${ }^{b}$ Research Institute of Nephrology, Zhengzhou University, Zhengzhou, China; `Key Laboratory of Precision \\ Diagnosis and Treatment for Chronic Kidney Disease in Henan Province, Zhengzhou, China; ${ }^{d}$ Core Unit of \\ National Clinical Medical Research Center of Kidney Disease, Zhengzhou, China; 'Department of Renal Pathology, \\ The First Affiliated Hospital of Zhengzhou University, Zhengzhou, China
}

\section{Keywords}

$\lg$ A nephropathy $\cdot$ Mucosal immunity $\cdot$ Secretory $\lg A \cdot$

Mesangial-tubular crosstalk

\section{Abstract}

Background: IgA nephropathy (IgAN) is characterized by the mesangial deposition of pathogenic IgA. We previously detected the deposition of pathogenic secretory $\lg \mathrm{A}(\mathrm{S} \lg \mathrm{A})$ in the mesangium of about one-third of IgAN patients. Tubulointerstitial injury has an important role in the development of IgAN. However, the relationship between SIgA and tubulointerstitial damage is currently unclear. In this work, the role of the mesangial-tubular crosstalk was explored in the tubulointerstitial damage in SlgA-induced IgAN. Methods: SIgA deposition in renal tissues of IgAN patients was detected by immunofluorescence. Flow cytometry was used to assess the binding of SIgA to human renal mesangial cells (HRMC) and human proximal tubule epithelial (HK-2) cells. HK-2 was co-cultured with HRMC added with SIgA isolated from patients or normal volunteers. Protein synthesis and gene expressions of TNF- $\alpha$, TGF- $\beta 1$, and MCP- 1 were determined by ELISA and PCR, respectively. The expressions of the above cytokines in renal tissues of patients and normal controls were detected by immunohistochemistry. Results: Twenty-nine of 96 patients had SIgA deposition in the mesangium, but SIgA was rarely detected in the tubulointerstitium. The binding rate of SIgA to HK-2 (2.79\%) was significantly lower than that of HRMC (81.6\%) $(p<0.001)$. The expressions of TNF- $\alpha$, TGF- $\beta 1$, and MCP-1 in HRMC were significantly higher than in SIgA-stimulated HK-2 $(p<0.05)$, and their expressions were significantly higher in the SIgAstimulated co-culture group compared with SIgA-stimulated HRMC $(p<0.05)$. The expressions of the above cytokines were mainly detected in tubulointerstitium of IgAN patients with positive and negative SIgA deposition, without significant difference between the 2 groups, but to a significantly higher level than that in normal controls, and their expressions positively correlated with tubulointerstitial injury. Conclusion: Inflammatory factors released from the mesangium after SIgA deposition might mediate tubulointerstitial damage via mesangial-tubular crosstalk in IgAN.

(C) 2021 The Author(s)

Published by S. Karger AG, Basel

Junjun Zhang and Ruwen Zhou equally contributed to this work. karger@karger.com www.karger.com/kbr

Karger $\stackrel{\text { ' }}{=}$
(C) 2021 The Author(s)

Published by S. Karger AG, Basel

This article is licensed under the Creative Commons AttributionNonCommercial-NoDerivatives 4.0 International License (CC BYNC-ND) (http://www.karger.com/Services/OpenAccessLicense) Usage and distribution for commercial purposes as well as any distribution of modified material requires written permission.
Correspondence to:

Junjun Zhang, fcczhangjj1 @zzu.edu.cn

Zhangsuo Liu, zhangsuoliu @ zzu.edu.cn 


\section{Introduction}

IgA nephropathy (IgAN), a common primary glomerular disease with a complex pathological mechanism, is the leading cause of kidney failure. In China, the incidence of IgAN is approximately $45.3 \%$ in those with primary glomerulonephritis [1], 30-40\% of patients with IgAN progress to renal failure within $10-25$ years [2]. Currently, it is thought that aberrant glycosylated IgA, genetic factors, and mucosal immunity are involved in the pathogenesis of IgAN [3,4], but the exact pathogenesis remains uncertain. Recently, many studies have shown that mucosal immunity, especially antibody-secretory IgA (SIgA), has a vital role in IgAN [57]. Our previous research also confirmed that SIgA deposited in the mesangium was pathogenic in IgAN patients. SIgA stimulated the proliferation and activation of mesangial cells to release various cytokines and participated in kidney damage [8]. Therefore, mucosal immunity, especially SIgA, plays a crucial role in the development of IgAN.

IgAN was previously considered a mesangial proliferative glomerular disease, but studies suggested that tubulointerstitial damage correlated more closely with disease progression than glomerular injury $[9,10]$. A subgroup of IgAN with severe tubulointerstitial damage usually progress faster to kidney failure [11]. The severity of tubulointerstitial damage is related to decrease renal function and long-term prognosis of glomerular diseases including IgAN, and tubular atrophy/interstitial fibrosis is an independent risk predictor of disease progression in IgAN patients [11-15]. Previous studies showed that tumour necrosis factor- $\alpha$ (TNF- $\alpha$ ) secreted from mesangial cells after IgA deposition activated renal tubular cells via glomerulo-tubular communication [16] and IgA-activated mesangial cells secreted aldosterone and angiotensin II, which regulated oxidative damage and induced apoptosis in tubular epithelial cells via glomerulo-tubular communication [17]. However, the mechanisms related to how SIgA mediates IgAN tubulointerstitial damage are unclear. We previously documented that approximately one-third of IgAN patients had SIgA deposition in the mesangium [8]. Therefore, we hypothesized that SIgA mediates tubulointerstitial damage in IgAN through mesangial-tubular crosstalk as IgA. Here, we investigated SIgA deposition in the tubulointerstitium and its binding to tubular cells with a focus on the effects of inflammatory regulators released from the mesangium triggered by SIgA deposition on tubular cells.

Mechanism of Secretory IgA in

Tubulointerstitial Injury in IgAN

\section{Materials and Methods}

\section{Patient Samples}

Ninety-six patients with renal-biopsy-proven IgAN, from the Renal Division, First Affiliated Hospital of Zhengzhou University from September 2017 to September 2018, were enrolled in this study. We excluded patients with systemic lupus erythematosus, Henoch Schönlein purpura, active hepatitis or liver cirrhosis, diabetes mellitus, or secondary IgAN-related diseases. The clinical data of all IgAN patients were collected before renal biopsy. Ten cases of normal renal tissue adjacent to kidney cancer were recruited as normal controls.

\section{Detection of SIgA Deposition by Immunofluorescence}

Immunofluorescence was used to detect the deposition of SIgA in the glomerulus and tubulointerstitial area of IgAN patients. Sections $(4 \mu \mathrm{m})$ were excised from frozen kidney biopsy tissues, fixed in cold acetone for $10 \mathrm{~min}$, blocked with $5 \%$ bovine serum albu$\mathrm{min} / \mathrm{PBS}$ (BSA/PBS) for $30 \mathrm{~min}$, then incubated with 5\% BSA/PBS 1:100 diluted mouse monoclonal anti-human secretory component (SC) (Abcam, Cambridge, UK) overnight at $4^{\circ} \mathrm{C}$, PBS was used instead of the mouse monoclonal anti-human SC as the negative control. Next, 5\% BSA/PBS 1:200 diluted Alexa Fluor 594-labelled donkey anti-mouse IgG (Invitrogen, Paisley, UK) and 1:30 diluted fluorescein isothiocyanate-labelled rabbit anti-human IgA (Dako, Glostrup, Denmark) were added and incubated at $37^{\circ} \mathrm{C}$ for $60 \mathrm{~min}$ in the dark. All sections were observed by fluorescence microscopy (DFC 7000T; Leica, Wetzlar, Germany).

\section{Collection of Saliva}

A saliva sample from IgAN patients with SIgA deposition and volunteers (healthy controls) who were age and gender matched, without history of kidney disease, hepatitis, and recent mucosal infection, was collected in the morning as previously described [8]. After centrifugation, the supernatants of saliva were collected for the isolation and purification of SIgA. Before collecting specimens, written informed consent was signed by all patients and healthy subjects.

\section{Isolation of SIgA}

Jacalin affinity chromatography (Pierce Chemical Company, Rockford, IL, USA) was used to isolate and purify SIgA as previously described [8]. Briefly, saliva diluted 1:1 with $0.01 \mathrm{M}$ PBS buffer was applied to a prepared jacalin column. The column was washed with $0.01 \mathrm{M}$ PBS until the optical density at $280 \mathrm{~nm}$ was $<0.1$, and then SIgA was eluted with melibiose (Sigma, St. Louis, MO, USA) in $0.01 \mathrm{M}$ PBS until the optical density returned to 0.1 . The eluted fractions were collected and concentrated using an ultrafiltration centrifuge tube (Millipore, Bedford, MA, USA). The concentrated sample was dialyzed against $0.01 \mathrm{M}$ PBS ( $\mathrm{pH}$ 7.4) for $24 \mathrm{~h}$ to remove melibiose. A sandwich ELISA was used to detect the concentration of the purified SIgA. In brief, polystyrene microtiter plates (Costar, Mankato, MN, USA) were coated with 5.1 $\mu \mathrm{g} / \mathrm{mL}$ mouse monoclonal anti-human SC (Sigma, Saint Louis, MO, USA) in carbonate buffer (pH 9.6) (100 $\mu \mathrm{L} /$ well) and incubated overnight at RT. Then, after washing with PBS/0.1\% Tween 20 (PBST), the plates were blocked with PBST/1\% BSA (PBST/ BSA). Subsequently, the plates were incubated with a purified SIgA solution ( $1: 100$ dilution) at $37^{\circ} \mathrm{C}$ for $1 \mathrm{~h} .1 \% \mathrm{BSA} / \mathrm{PBST}$ was used as the blank control; 1\% BSA/PBST diluted human SIgA (0.625 g/ $\mathrm{mL}$ ) was used as the positive control. After washing, rabbit poly- 
clonal anti-human IgA (Dako, Glostrup, Denmark) (1:5,000 dilution) was added, and then the plates were incubated with horseradish peroxidase-conjugated goat anti-rabbit IgG (Zhongshan Biotech, Beijing, China) $\left(1: 10,000\right.$ dilution) at $37^{\circ} \mathrm{C}$ for $1 \mathrm{~h}$. The reaction was revealed using $0.1 \mathrm{M}$ citrate phosphate buffer ( $\mathrm{pH}$ 5.0) containing $0.1 \% \mathrm{H}_{2} \mathrm{O}_{2}$ and $0.04 \%$ o-phenylene diamine and then was stopped by $1 \mathrm{~mol} / \mathrm{L} \mathrm{H}_{2} \mathrm{SO}_{4}$. Next, the optical density was measured at $490 \mathrm{~nm}$. SIgA purified and isolated from human colostrum (Serotec, Oxford, UK) was used to establish the standard curve, and the concentration of saliva SIgA was calculated.

\section{Flow Cytometry}

Flow cytometry was used to detect the binding rate of SIgA to human renal mesangial cells (HRMC) and human proximal tubule epithelial cells (HK-2). HRMC and HK-2 were harvested by using trypsin/EDTA for $2 \mathrm{~min}$ at $37^{\circ} \mathrm{C}$ when they were grown to log phase. Cell numbers were counted using a haemocytometer with a mean value of $1 \times 10^{5}$ cells per well. Staining was performed at $4^{\circ} \mathrm{C}$, and then the cells were incubated with SIgA (final concentration $400 \mu \mathrm{g} / \mathrm{mL}$ ) for $30 \mathrm{~min}$. After washing, the cells were incubated with $10 \mu \mathrm{L}$ of fluorescein isothiocyanate -conjugated goat anti-human IgA antibody. Background control staining was evaluated by the reaction with preimmune $\mathrm{F}\left(\mathrm{ab} \mathrm{b}^{\prime}\right) 2$ fragment of fluorescein-conjugated isotypic antibody. The BD FACS Calibur (Becton Dickinson, Holdrege, NE, USA) and Flowjo were used to analyse the stained cells (circled live cells). A minimum of 1,000 fixed cells was analysed for each specimen.

\section{Cell Culture and Treatments}

By culturing HRMC and HK-2 in vitro, we observed the differential expression of inflammatory factors in $\mathrm{HK}-2$ stimulated by SIgA alone and HK-2 co-cultured with HRMC stimulated by SIgA, so as to explore the mechanism of SIgA on HK-2. HRMC and mesangial cell culture medium (Cat. \#4201), foetal bovine serum (FBS) and supplements were obtained from ScienCell ${ }^{\mathrm{TM}}$ (Carlsbad, CA, USA). HRMCs were grown in mesangial cell culture medium supplemented with $2 \%$ mesangial cell growth supplement, penicillin $(100 \mathrm{U} / \mathrm{mL})$, streptomycin $(100 \mu \mathrm{g} / \mathrm{mL})$, and $5 \% \mathrm{FBS}$. The HK-2 cells which were kindly provided by professor Yang Li of Peking University First Hospital were grown in DMEM/F12 supplemented with penicillin $(50 \mathrm{U} / \mathrm{mL})$, streptomycin $(50 \mu \mathrm{g} / \mathrm{mL})$, and $10 \%$ FBS. Both cells were cultured in a $5 \% \mathrm{CO}_{2}, 95 \%$ air humidification incubator at $37^{\circ} \mathrm{C}$. After cell starvation for $24 \mathrm{~h}$ using medium without FBS to achieve synchronization, on the one hand, HRMC and HK-2 were seeded into 6 -well plates $\left(1 \times 10^{6}\right.$ cells per well $)$ and were stimulated with SIgA $(400 \mu \mathrm{g} / \mathrm{mL})$ for $24 \mathrm{~h}$, separately. On the other hand, HRMC and HK-2 were co-cultured as follows: HRMCs were seeded into a 6 -well plate $\left(1 \times 10^{6}\right.$ cells per well $)$ and SIgA $(400 \mu \mathrm{g} / \mathrm{mL})$ was added; then the transwell insert $(1.0 \mu \mathrm{m}$ well, Millipore, Burlington, MA, USA) was placed in the 6-well plate, and HK-2 cells were seeded into the transwell insert $\left(1 \times 10^{6}\right.$ cells per chamber) and incubated together for $24 \mathrm{~h}$. These soluble molecules, such as TNF- $\alpha$, TGF- $\beta 1$, and MCP- 1 , with molecular weights $<20 \mathrm{kDa}$, can freely pass through the transwell insert $(1.0 \mu \mathrm{m}$ well, Millipore, Burlington, MA, USA) between HRMC and HK-2 cells. This ensures that these 2 kinds of cells can interact with each other via soluble molecules during physiological separation. The drawing of HK-2 and HRMC co-culture is shown in online suppl. Fig. 1; see www.karger.com/doi/10.1159/000514183 for all online suppl. material.
Table 1. Primer sequences for real-time RT-PCR

\begin{tabular}{ll}
\hline Gene & Primer \\
\hline MCP-1 forward & 5'-CAGCCAGATGCAATCAATGCC-3' \\
MCP-1 reverse & 5'-TGGAATCCTGAACCCACTTCT-3' \\
TNF- $\alpha$ forward & 5'-CCTCTCTCTAATCAGCCCTCTG-3' \\
TNF- $\alpha$ reverse & 5'-GAGGACCTGGGAGTAGATGAG-3' \\
TGF- $\beta 1$ forward & 5'-CTAATGGTGGAAACCCACAACG-3' \\
TGF- $\beta 1$ reverse & 5'-TATCGCCAGGAATTGTTGCTG-3' \\
ACTB forward & 5'-CCTGGCACCCAGCACAAT-3' \\
ACTB reverse & 5'-GCTGATCCACATCTGCTGGAA-3' \\
\hline
\end{tabular}

ACTB, $\beta$-actin; TNF- $\alpha$, tumour necrosis factor- $\alpha$; TGF- $\beta 1$, transforming growth factor- $\beta 1$; MCP- 1 , monocyte chemoattractant protein-1.

\section{Enzyme-Linked Immunosorbent Assay}

To detect TNF- $\alpha$, transforming growth factor- $\beta 1$ (TGF- $\beta 1$ ) and monocyte chemotactic protein-1 (MCP-1) levels in cell culture supernatants, standard sandwich ELISAs were performed using human TNF- $\alpha$, TGF- $\beta 1$, and MCP-1 ELISA kits (R\&D Systems, Minneapolis, MN, USA), according to the manufacturer's specifications.

\section{Real-Time Quantitative PCR}

Total cellular RNA was prepared using TRIZOL ${ }^{\circledR}$ Reagent (Invitrogen, Carlsbad, CA, USA) and used for cDNA synthesis with a reverse transcription kit (Takara Bio, Shiga, Japan) according to the manufacturer's instructions. RT-qPCR was carried out by using SYBR Green PCR Master mix and was performed on a realtime quantitative PCR system (LightCycler ${ }^{\circledR} 480$ II; Roche). The relative mRNA expression of TNF- $\alpha$, TGF- $\beta 1$, and MCP- 1 was calculated by the $2^{-\Delta \Delta C T}$ method. The primer sequences used in this study are shown in Table 1.

\section{Immunohistochemistry}

The expressions of TNF- $\alpha$, TGF- $\beta 1$, and MCP- 1 in renal tissues were detected by immunohistochemistry, and their correlations with tubulointerstitial injury were analysed. Renal tubulointerstitial injury scores were assessed in 29 IgAN patients with SIgA deposition, 29 IgAN patients without SIgA deposition. Tubular atrophy, interstitial fibrosis, and interstitial inflammatory cell infiltration were scored as follows: (0) absent; $(1)<25 \%$ of the tubules and interstitium; (2) $25-50 \%$ of the tubules and interstitium; and (3) $>50 \%$ of the tubules and interstitium. The sum of these scores resulted in a final score for tubulointerstitial lesions of $0-9$. These patients were divided into 3 groups: mild lesions (0-2), moderate lesions (3-5), and severe lesions (6-9) [18]. Immunohistochemistry was performed to detect TNF- $\alpha$, TGF- $\beta 1$, and MCP- 1 on renal biopsies of different severities of IgAN patients with positive SIgA deposition, negative SIgA deposition, and normal controls. Paraffin sections $(3 \mu \mathrm{m})$ were dewaxed, hydrated, treated for antigen retrieval, blocked for endogenous peroxidase activity, and then incubated with primary antibodies overnight at $4^{\circ} \mathrm{C}$. After washing, biotinylated secondary antibody (1:100 dilution, Zhongshan Jinqiao, Beijing, China) was incubated with the sections at $37^{\circ} \mathrm{C}$ for $30 \mathrm{~min}$. Colour development was performed with diaminobenzi- 

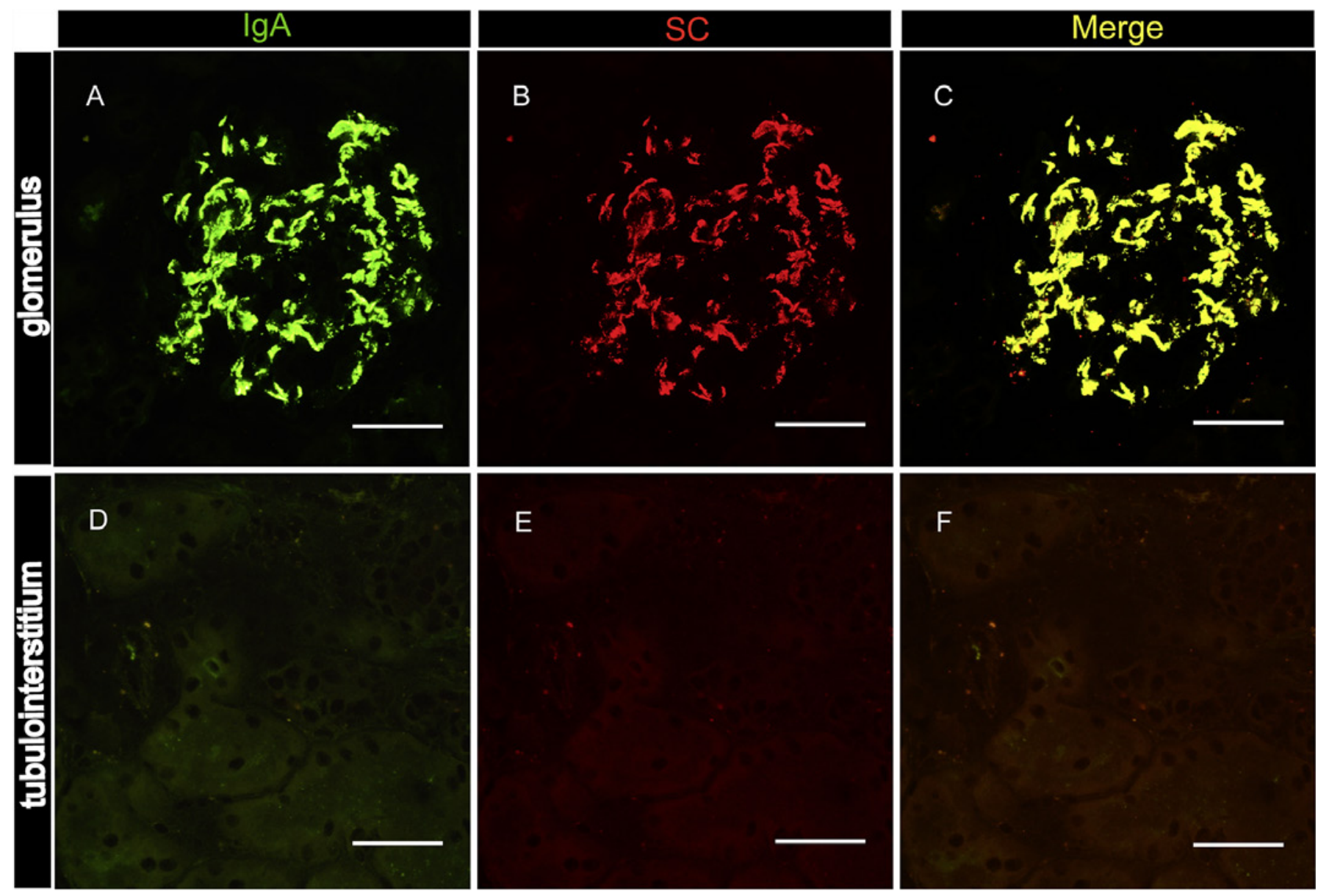

Fig. 1. Representative images of the colocalization of SC and IgA in renal tissues of IgAN patients detected by immunofluorescence. Double staining of IgA (green) and SC (red) shows nearly complete colocalization (yellow) in glomeruli $(\mathbf{A}-\mathbf{C})$ and no significant signal in the tubulointerstitial areas (D-F). Scale bar, $50 \mu \mathrm{m}$. SC, secretory component.

Table 2. Information of antibodies used in this study

\begin{tabular}{llll}
\hline Antibody & IHC & Specificity & Company \\
\hline TNF- $\alpha$ & $1: 100$ & Rabbit polyclonal & Abcam, Cambridge, UK \\
TGF- $\beta 1$ & $1: 100$ & Rabbit polyclonal & Abcam, Cambridge, UK \\
MCP-1 & $1: 100$ & Rabbit polyclonal & Abcam, Cambridge, UK \\
\hline
\end{tabular}

TNF- $\alpha$, tumour necrosis factor- $\alpha$; TGF- $\beta 1$, transforming growth factor- $\beta 1$; MCP-1, monocyte chemoattractant protein- 1 .

dine. Finally, the sections were counterstained with haematoxylin, dehydrated, sealed, and observed by light microscopy. The areas containing brown granules were independently counted by 3 pathologists who were blinded to the clinical parameters, and the samples were scored according to the proportion of positive cells as follows: none $=0 ; 1-25 \%=1 ; 26-50 \%=2 ; 51-75 \%=3$; and $\geq 76 \%=4$. The staining intensity was scored as 0 (negative), 1 (weak), 2 (moderate), and 3 (strong). The final histological (h) scores ( $0-12)$ were determined by multiplying the 2 subscores, and $\mathrm{h}$ values of $0-3,4-6,7-9$, and $9-12$ were classified as negative $(\mathrm{N})$, low positive $(+)$, medium positive $(++)$, and strongly positive $(+++)$, respectively [19]. Detailed information on the primary antibodies used in this study is listed in Table 2.

\section{Statistical Analysis}

Data with normal distributions were given as the mean $\pm \mathrm{SD}$ and analyzed with an independent-sample $t$ test. Non-normally distributed variables were presented as median and interquartile range and compared by the Mann-Whitney $U$ test. Categorical data were summarized by percentages. Spearman's rank correlation coefficient was used to test the correlation of renal tissue expressions of TNF- $\alpha$, TGF- $\beta 1$, and MCP- 1 with tubulointerstitial injury scores, and these are presented as scatterplots. A value of $p<0.05$ was considered statistically significant. Analysis was performed with SPSS version 20.0 (SPSS Inc., Chicago, IL, USA) and GraphPad Prism 6 software (GraphPad Software, Inc., La Jolla, CA, USA). 
Table 3. Demographic and clinical characteristics of patients with and without SIgA deposition in the kidneys.

\begin{tabular}{lccc}
\hline & $\begin{array}{l}\text { SIgA }(+) \\
(n=29)\end{array}$ & $\begin{array}{l}\text { SIgA (-) } \\
(n=67)\end{array}$ & $p$ value \\
\hline Gender (male/female) & $17 / 12$ & $42 / 25$ & 0.707 \\
Age, years & $31(25-40)$ & $30(24-39)$ & 0.652 \\
Infection history (positive/negative) & $7 / 22$ & $17 / 50$ & 0.898 \\
Haematuria (positive/negative) & $5 / 24$ & $7 / 60$ & 0.355 \\
SBP, mm Hg & $134(123-148)$ & $131(117-145)$ & 0.526 \\
DBP, mm Hg & $90(82-99)$ & $89(80-100)$ & 0.415 \\
Haemoglobin, g/L & $130.207 \pm 22.158$ & $129.410 \pm 21.527$ & 0.869 \\
ALB, g/L & $39.9(33-41.35)$ & $39.3(32.3-43.3)$ & 0.886 \\
SCr, mmol/L & $76(62.5-131)$ & $79(65-120)$ & 0.889 \\
UA, mmol/L & $333(244-476)$ & $332(213-401.5)$ & 0.159 \\
eGFR, mL/min & $83.49(64.93-109.61)$ & $88.82(56.31-108.53)$ & 0.669 \\
Proteinuria, g/24h & $1.35(0.52-3.02)$ & $1.58(0.93-3.15)$ & 0.311 \\
Cystatin C, mg/L & $0.96(0.80-1.57)$ & $1.00(0.89-1.65)$ & 0.375 \\
$\beta 2-M G, \mathrm{mg} / \mathrm{L}$ & $1.83(1.33-3.22)$ & $2.24(1.64-3.40)$ & 0.206 \\
\hline
\end{tabular}

The normally distributed data are expressed as mean \pm SD, while the skewed data are expressed as median and range. A value of $p<0.05$ was considered statistically significant. SIgA (+), patients with SIgA deposition in the kidneys; SIgA (-), patients without SIgA deposition in the kidneys; SBP, systolic blood pressure; DBP, diastolic blood pressure; ALB, serum albumin; SCr, serum Cr; UA, uric acid; eGFR, estimated glomerular filtration rate; $\beta 2-\mathrm{MG}, \beta 2$ microglobulin; SIgA, secretory IgA.

\section{Results}

\section{SIgA Deposition in the Kidney of IgAN Patients}

Overall, 96 patients were included in the study. Renal deposition of IgA and SC were colocalized in these patients as assessed by fluorescence microscopy (Fig. 1). Mesangial IgA deposition was detected in all IgAN patients. Significant mesangial area SC deposits were detected in 29 patients (30.21\%) (Fig. 1A-C). No significant deposition of IgA and SC was observed in the renal tubular area (Fig. 1D-F). In the negative control group, only the distribution of green IgA without SC deposition in the mesangial area was observed (online suppl. Fig. 2A), and no green IgA and red SC deposition in tubulointerstitial area were found (online suppl. Fig. 2B). There was no deposition of SC or IgA in glomerulus or tubulointerstitium of the healthy control group (normal renal tissues adjacent to kidney cancer) (online suppl. Fig. 3).

\section{Associations between SIgA Deposition and Clinical \\ Parameters}

The clinical parameters between the patients with SIgA deposition and without SIgA deposition in the kidneys were compared in Table 3 . There were no significant differences in the incidence of infection history and haematuria, levels of hypertension, haemoglobin, serum al- bumin, serum Cr, uric acid, eGFR and proteinuria/24 h between the patients with SIgA deposition and without SIgA deposition in the kidneys. No significant differences were detected in the levels of serum cystatin $\mathrm{C}$ and $\beta 2 \mathrm{mi}-$ croglobulin between the 2 groups.

\section{Binding Rate of SIgA to HRMC and HK-2}

Although SIgA was deposited in the glomerular mesangium, there was no significant presence of SIgA in the tubular area. Figure $2 \mathrm{~A}$ and $\mathrm{B}$ show the binding rate of SIgA to HRMC and HK-2 cells, respectively, as determined by flow cytometry. The binding rate of SIgA from IgAN patients to either cell type was higher than the binding rate of SIgA from normal control subjects (HK-2: P-SIgA vs. N-SIgA, $p<0.001$; HRMC: P-SIgA vs. N-SIgA, $p<0.001)$. However, under the same conditions, the ability of HK-2 to bind to SIgA was significantly lower than that of HRMC for patients and healthy controls (P-SIgA: HK-2 vs. HRMC, $p<0.001$; N-SIgA: HK-2 vs. HRMC, $p<0.001)$.

\section{Expressions of TNF- $\alpha, T G F-\beta 1$, and MCP-1 in HK-2}

Cultured with SIgA-Stimulated HRMC

Following the histological finding of the non-deposition of SIgA in tubular areas and infrequent binding of SIgA to HK-2, we performed an in vitro co-culture ex- 


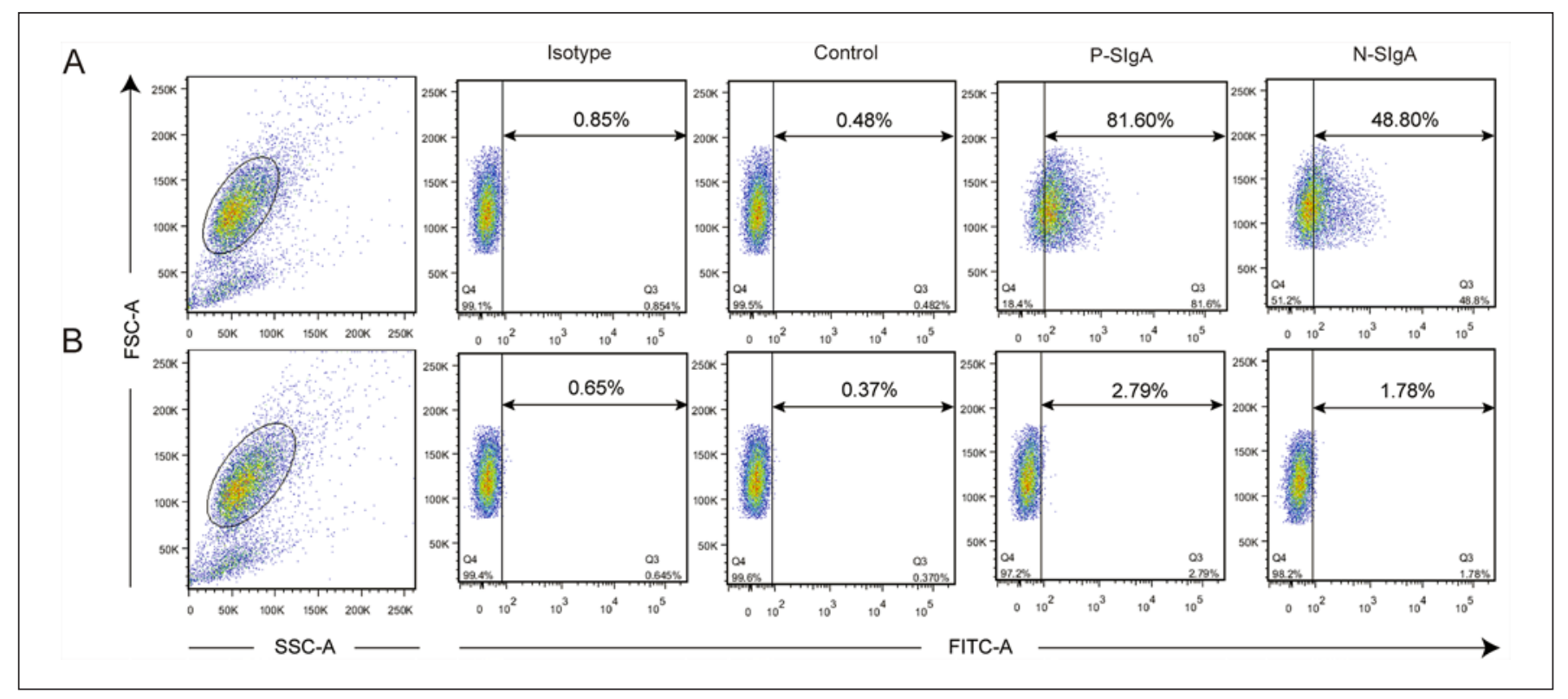

Fig. 2. Representative images of SIgA binding to HRMC and HK-2 determined by flow cytometry. Binding of SIgA to (A) HRMC or HK-2 (B). The binding rate of P-SIgA to HRMC and HK-2 was increased compared with N-SIgA, while the binding of P-SIgA and $\mathrm{N}$-SIgA to HK-2 was significantly lower than that of HRMC. Con-

periment to investigate whether SIgA might modulate tubulointerstitial damage of IgAN via mesangial-tubular interactions. No obvious changes in the protein synthesis and gene expression of TNF- $\alpha$, TGF- $\beta 1$, and MCP- 1 were observed in HK-2 stimulated by SIgA from patients or healthy subjects. However, protein release (TNF- $\alpha$ : $p=$ 0.001 ; TGF- $\beta 1: p<0.001$; MCP- $1: p=0.002)$ and mRNA expression (TNF- $\alpha$ : $p<0.001$; TGF- $\beta 1: p=0.001$; MCP-1: $p<0.001$ ) of these cytokines from HRMC stimulated by SIgA from patients were significantly higher than that from healthy controls. After coculturing HK-2 with HRMC added with SIgA, the protein synthesis of TNF- $\alpha$, TGF- $\beta 1$, and MCP- 1 in the co-culture group was significantly higher than that in the SIgA-stimulated HRMC group (TNF- $\alpha$ : $p<0.001$; TGF- $\beta 1: p<0.001$; MCP- $1: p=$ 0.002 ) (Fig. $3 \mathrm{~A}$ ), suggesting that there may be a communication between HRMC and HK-2 which upregulated the expression of these inflammatory factors. Similar findings were observed for the gene expressions of TNF- $\alpha$, TGF- $\beta 1$, and MCP-1. The mRNA expressions of these cytokines in HK-2 and HRMC of the co-culture group stimulated by SIgA from patients were significantly higher than that in normal controls (co-HK-2, TNF- $\alpha$ : $p=$ 0.027 ; TGF- $\beta 1: p=0.023$; MCP- $1: p=0.020$; co-HRMC, $p<0.001)$. The gene expressions of TNF- $\alpha$, TGF- $\beta 1$, and

Mechanism of Secretory IgA in

Tubulointerstitial Injury in IgAN trol, no stimulation added; P-SIgA, SIgA purified from IgAN patients; N-SIgA, SIgA purified from healthy subjects; SIgA, secretory IgA; HRMC, human renal mesangial cell; HK-2, human proximal tubule epithelial cell.

MCP-1 in HK-2 and HRMC of the co-culture group were significantly higher than those in HK-2 or HRMC stimulated by SIgA alone (co-HK-2 vs. HK-2, TNF- $\alpha$ : $p=0.006$; TGF- $\beta 1: p=0.002$; MCP- $1: p=0.006$; co-HRMC vs. HRMC, TNF- $\alpha: p=0.015$; TGF- $\beta 1: p=0.038$; MCP- 1 : $p=0.010)$ (Fig. 3B).

\section{Histologic Assessment of Glomerular and Tubular Damage Induced by Cytokines as Measured by \\ Immunohistochemistry Staining}

The results of immunohistochemistry showed that TNF- $\alpha$, TGF- $\beta 1$, and MCP- 1 were mainly expressed in the tubulointerstitium of IgAN patients both with SIgA deposition and without SIgA deposition, and to a significantly higher level than that in normal controls, but there was no significant difference between the positive and negative SIgA deposition groups. Furthermore, the expressions of these factors were increased with an increase of the degree of tubulointerstitial injury (Fig. 4A). The proportion of high histological scores for TNF- $\alpha$, TGF- $\beta 1$, and MCP-1 gradually increased with the exacerbation of tubulointerstitial injury (Fig. 4B). Furthermore, the expressions of TNF- $\alpha$, TGF- $\beta 1$, and MCP-1 were positively correlated with tubulointerstitial injury (Fig. 4C). 


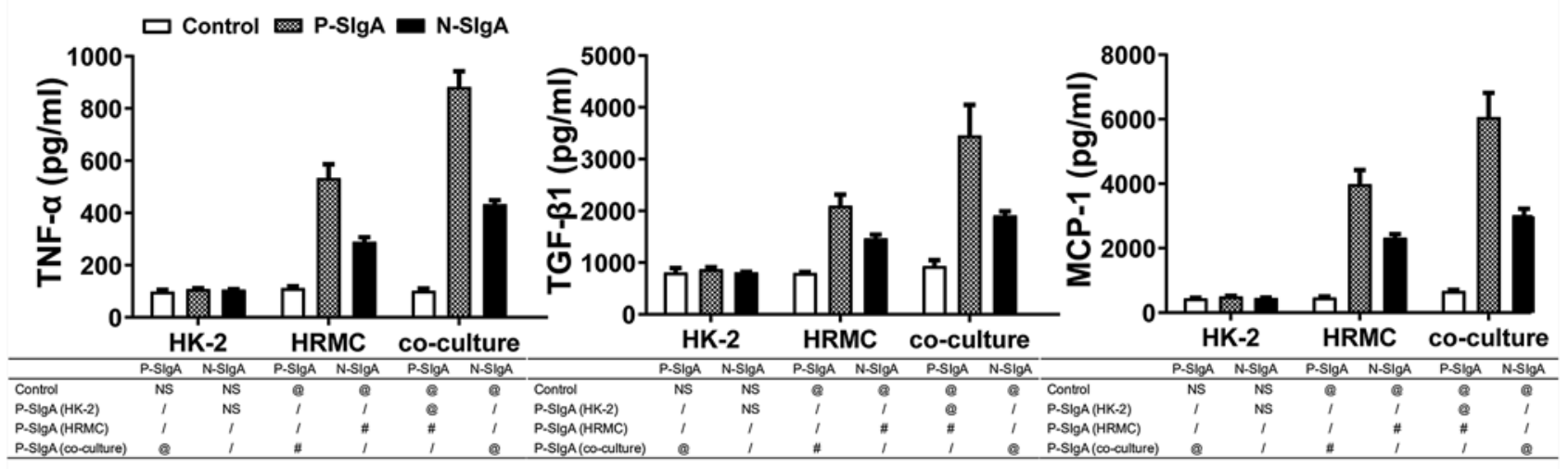

B

\section{mRNA expression (fold change)}
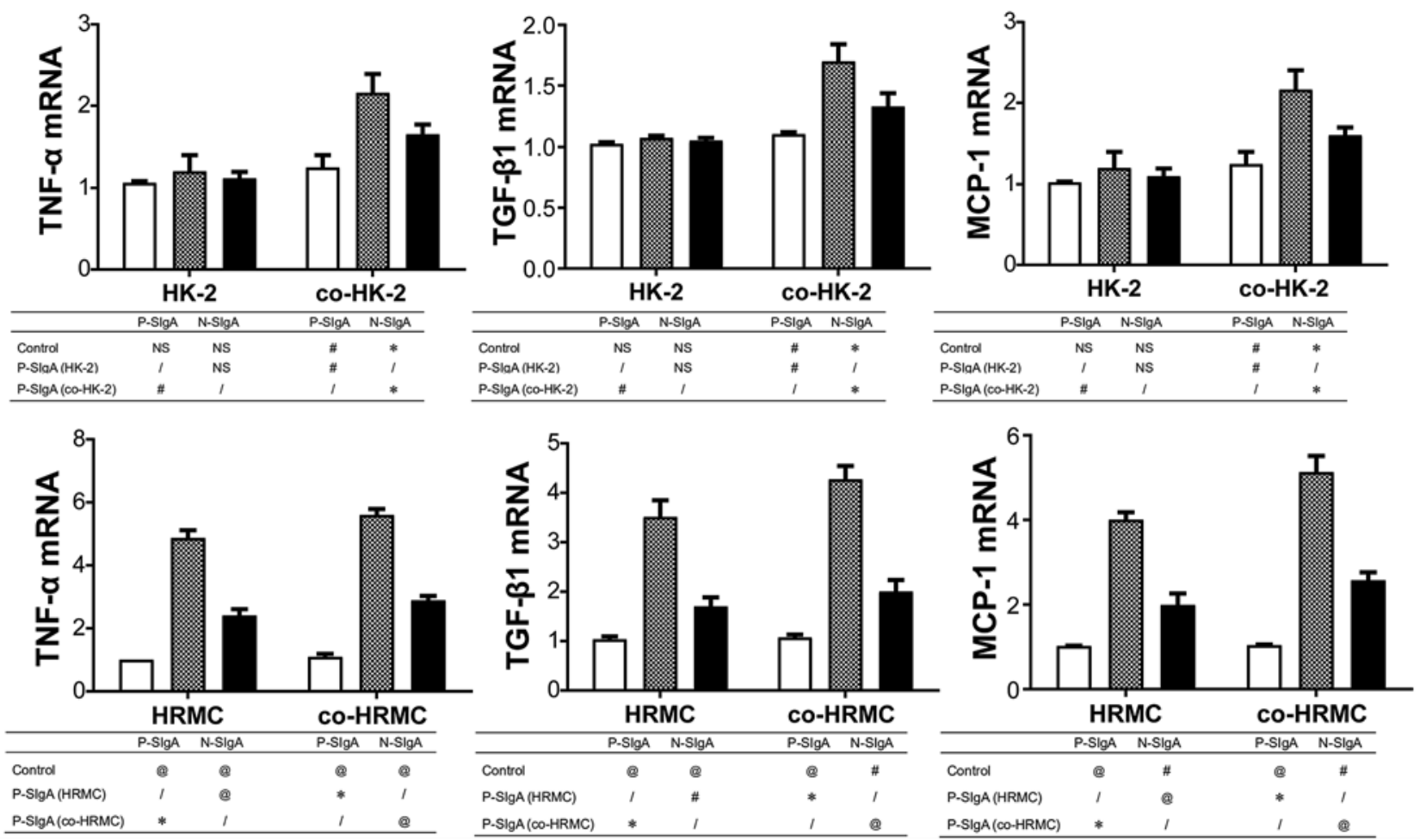

Fig. 3. Altered expression of TNF- $\alpha$, TGF- $\beta 1$, and MCP-1 in HK-2, HRMC, and co-culture groups after SIgA stimulation. A Protein synthesis of TNF- $\alpha$, TGF- $\beta 1$, and MCP- 1 in HK-2, HRMC, and co-culture groups stimulated by SIgA. B Gene expression of TNF- $\alpha$, TGF- $\beta 1$, and MCP- 1 in HK-2, co-HK-2, HRMC, and coHRMC stimulated by SIgA. The table below the graph depicts statistical analysis between groups using independent-sample $t$ test. Data represent the mean \pm SD from 3 independent experiments $\left({ }^{*} p<0.05,{ }^{\#} p<0.01,{ }^{@} p<0.001, \mathrm{NS}: p>0.05\right)$. Control, no stimulation added; P-SIgA, SIgA purified from IgAN patient; N-SIgA, SIgA purified from healthy subjects; co-culture, co-cultivation of HK-2 and HRMC; co-HK-2, HK-2 of co-culture groups; coHRMC, HRMC of co-culture groups; SIgA, secretory IgA; HRMC, human renal mesangial cell; HK-2, human proximal tubule epithelial cell. 
A Control
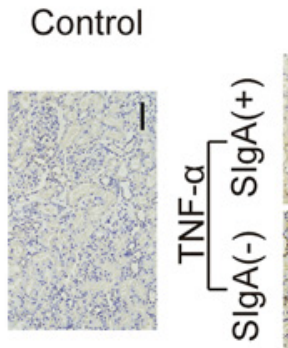

Group1 Group2 Group3
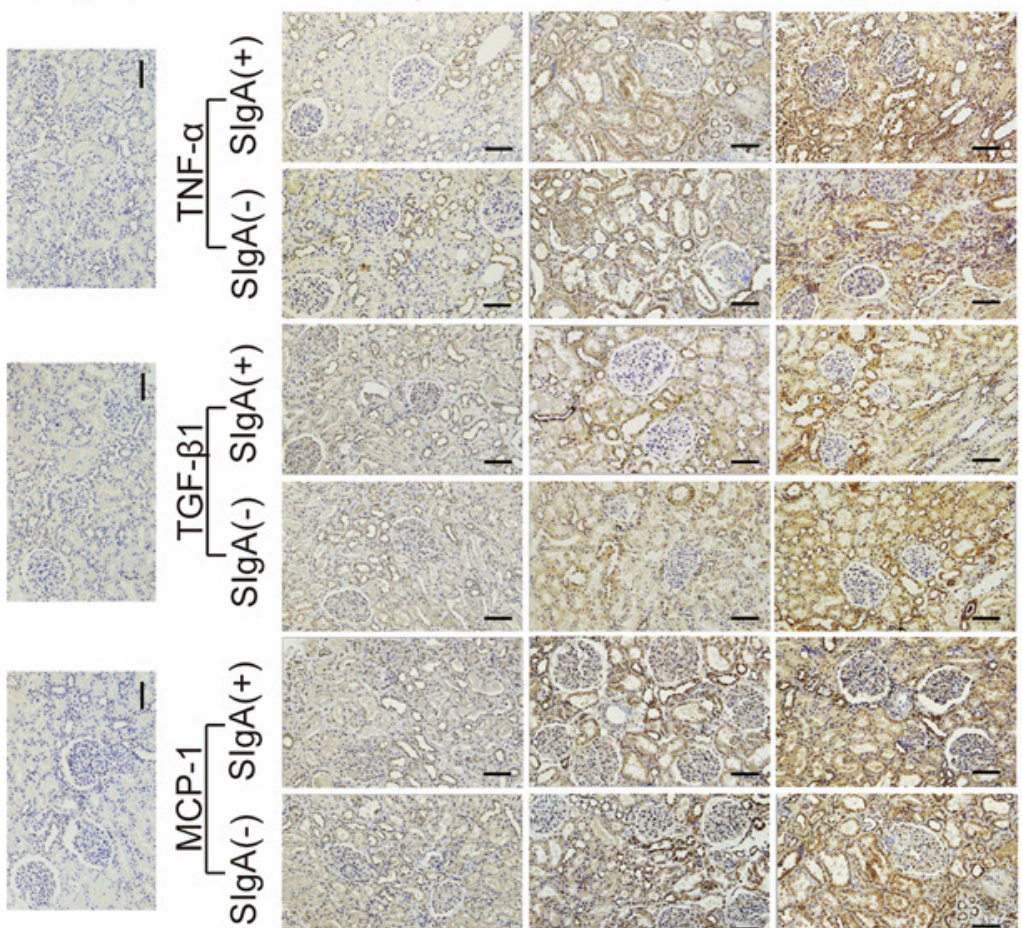

C
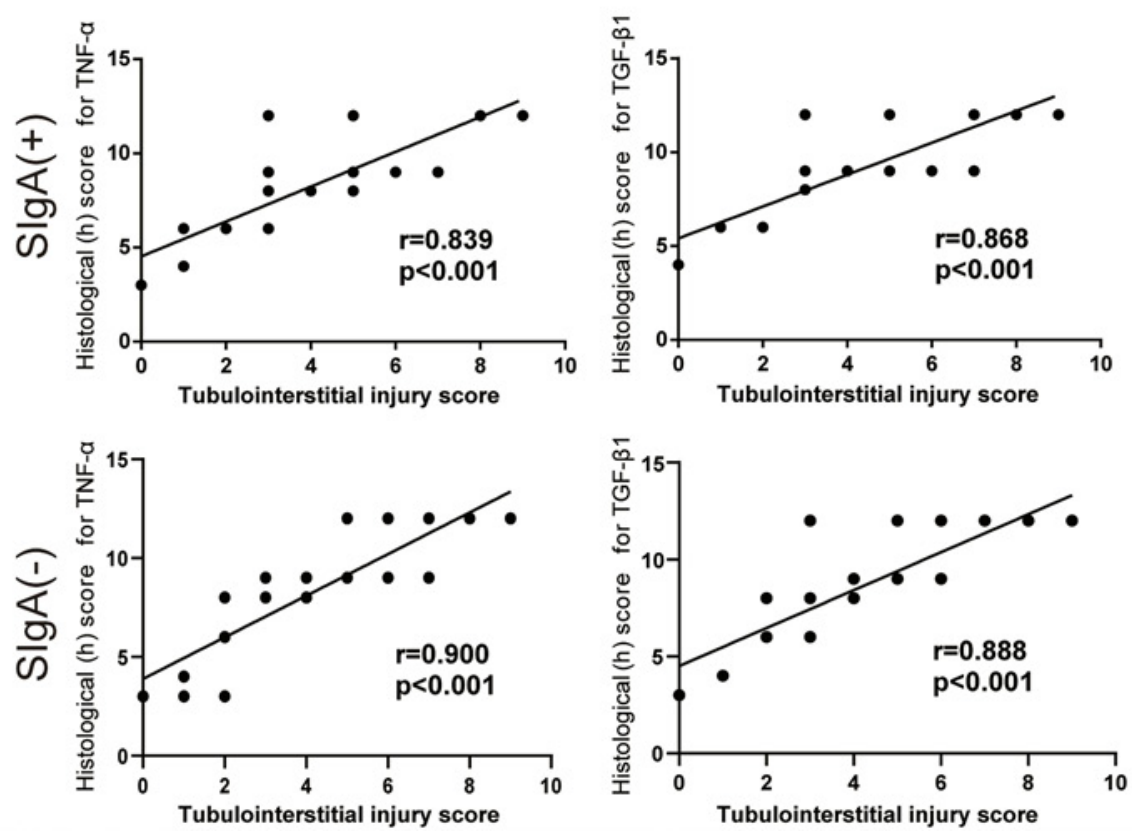

Tubulointerstitial injury score
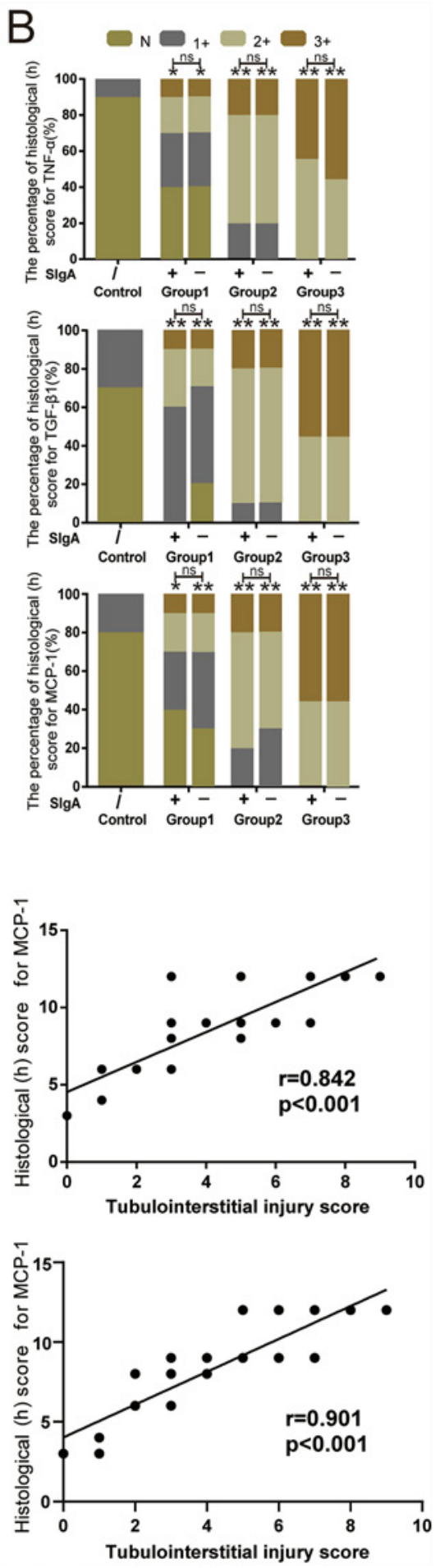

(For legend see next page.)

Mechanism of Secretory IgA in Tubulointerstitial Injury in IgAN
Kidney Blood Press Res 2021;46:286-297 DOI: $10.1159 / 000514183$ 


\section{Discussion}

IgAN is mainly characterized by glomerular injury in the early stages of disease, and as it progresses, it is often accompanied by tubulointerstitial lesions of various severities. The severity of tubulointerstitial damage was reported to be closely related to the progression of IgAN [9, 10]. However, the mechanism involved in tubulointerstitial damage in IgAN is poorly understood. Previous studies reported that "glomerulo-tubular communications" are important in glomerular and tubulointerstitial damage. Furthermore, immune complexes containing IgA promoted the release of inflammatory factors such as TNF- $\alpha$, TGF- $\beta 1$, IL- 6 , and AngII following the activation of HRMC, which induced further tubulointerstitial damage via glomerulo-tubular crosstalk [16, 17, 20-22]. Therefore, glomerulo-tubular crosstalk might be associated with the tubulointerstitial damage observed in IgAN.

Recently, the roles of mucosal immunity and SIgA have gained increasing attention in the pathogenesis of IgAN. SIgA, an antibody involved in human mucosal immunity, is primarily produced by B cells in mucosal lymphoid tissues and is distributed throughout the oral cavity, respiratory mucus, intestinal digestive fluid, and colostrum [23, 24], and a small amount can be returned to the blood. Our previous study suggested that serum macromolecular IgA in IgAN patients contained a large amount of SIgA, and that high SIgA deposition was present in the mesangial area of about one-third of all IgAN patients [6]. Furthermore, SIgA-induced HRMC proliferation and the secretion of IL-6, IL-8, MCP-1, TGF- $\beta 1$, and other inflammatory factors involved in renal damage [8]. We found that SIgA regulated the release of IL-6, IL8 , and IL- $1 \beta$ from HRMC by miR-100, miR-16-2-3p, and miR-100-3p, respectively [25]. Taken together, these findings indicate that SIgA has an important role in the development of IgAN.

Previous studies focused on the role of SIgA on mesangial cells, but the role of SIgA in IgAN tubulointerstitial damage has rarely been reported. IgA was reported to be mainly deposited in the mesangial area but was extremely rare in the tubulointerstitial area [16]. However, whether SIgA is deposited in the tubulointerstitial region is unclear. To investigate this, immunofluorescence was used to determine the expression of SIgA in the mesangial and tubular regions. About $30 \%$ of patients with IgAN had SIgA deposition in the mesangial area of renal tissues, but it was not expressed in the tubulointerstitium. We used flow cytometry to demonstrate the low binding rate of SIgA to HK-2 (2.79\%) and the high binding rate to HRMC (81.6\%). Previous studies reported that HRMC expressed surface IgA receptors including IgA Fc receptor (FCAR), asialoglycoprotein receptor (ASGR), polymeric immunoglobulin receptor (pIgR), $\mathrm{Fc} \alpha / \mu \mathrm{R}$, and transferrin receptor (TfR). However, HK-2 did not express mRNA for any known IgA receptors except for a small amount of transferrin receptor [16]. Another study reported that the transferrin receptor did not bind to SIgA [26]. These findings might explain why SIgA cannot be combined with HK-2. However, in our experiments, a $2.79 \%$ binding rate of SIgA to HK-2 does not exclude the possibility of nonspecific binding, but the specific mechanism needs further exploration.

Based on the above experimental results, our hypothesis was that SIgA did not directly act on HK-2 but might induce further damage by deposition in the mesangial area and/or inducing HRMC to secrete specific inflammatory mediators. To investigate this, we performed in vitro cell experiments. A transwell chamber was placed in a 6-well plate to simulate the physiological conditions of mesangial cells and renal tubular epithelial cells in vivo. This ensured that there was no direct communication between HRMC and HK-2 cells but that HRMC-HK-2 communication could occur via the secretion of regulatory factors. The expressions of inflammatory factors in HRMC, HK-2, and co-culture groups stimulated by SIgA were measured. Protein synthesis and gene expressions of TNF- $\alpha$, TGF- $\beta 1$ and MCP- 1 were upregulated in the
Fig. 4. Histologic assessment of renal injury by TNF- $\alpha$, TGF- $\beta 1$, and MCP-1 immunostaining in kidneybiopsies. A TNF- $\alpha$, TGF- $\beta 1$, and MCP-1 protein expression in adjacent normal tissues and renal tissues of SIgA (+) and SIgA (-) IgAN patients with different degrees of tubulointerstitial injury (scale bar, $100 \mu \mathrm{m}, \times 200 \mathrm{mag}$ nification). B Distribution of differentially expressed TNF- $\alpha$, TGF- $\beta 1$, and MCP-1 in adjacent normal tissues and renal tissues of IgAN patients with different degrees of injury was quantified. c Correlation between the histological (h) score of TNF- $\alpha$, TGF- $\beta 1$, and MCP-1 and the tubulointerstitial injury score. $(\mathrm{N})$ : negative $(0-3),(1+)$ : low positive (4-6), (2+): medium positive (7-9), and (3+): strongly positive $(9-12) .{ }^{*} p<0.05,{ }^{* *} p<0.01,{ }^{* * *} p<0.001$, compared with control, ns, not significant. SIgA (+), positive SIgA deposition; SIgA (-), negative SIgA deposition; Control, normal renal tissue; Group 1, patients with mild lesions; Group 2, patients with moderate lesions; Group 3, patients with severe lesions; IgAN, IgA nephropathy; SIgA, secretory IgA; TNF- $\alpha$, tumour necrosis factor- $\alpha$. 
SIgA-stimulated HRMC group in line with our previous study reporting an upregulation of MCP- 1 and TGF- $\beta 1$ expression in SIgA-stimulated HRMC [8]. The gene expression and protein synthesis of these cytokines were not increased in SIgA-stimulated HK-2, suggesting that SIgA might have no direct effect on HK-2. However, after cocultivating the cell lines with SIgA, the expressions of TNF- $\alpha$, TGF- $\beta 1$, and MCP- 1 were significantly higher in the HRMC of co-culture group than in the HRMC stimulated by SIgA alone group. In addition, these factors were highly expressed in the HK-2 of co-culture group. These results indicate that the interaction between HRMC and HK-2 might increase the expression of inflammatory factors in mesangial cells and induced tubular cells to secrete these inflammatory factors. Consequently, mesangial-tubular crosstalk may have a critical role in the tubulointerstitial damage observed in SIgA-induced IgAN. In this study, we only found a superficial phenomenon that the HK-2 co-cultured with SIgA-stimulated HRMC could express more inflammatory factors, but we did not confirm which pathway the SIgA-stimulated HRMC had taken or through which mediator, and then how these mediators modulated the release of inflammatory factors by HK-2, thereby participating in IgAN tubulointerstitial damage. This is a limitation of this study. We will further investigate these specific mechanisms in the future.

Following the in vitro cell experiments, we examined the expression of TNF- $\alpha$, TGF- $\beta 1$, and MCP- 1 in the renal tissues of IgAN patients and normal controls, found that in IgAN patients with positive and negative SIgA deposition, TNF- $\alpha$, TGF- $\beta 1$, and MCP- 1 were mainly expressed in the tubulointerstitial area, and there was no significant difference between the 2 groups, but the expression of the above cytokines of the 2 groups was significantly higher than that of the normal control groups, and the expression of above inflammatory factors were positively correlated with the aggravation of tubulointerstitial injury. Analyzing this, we speculated that $\operatorname{SIgA}$ and $\operatorname{IgA}$ might have a similar function in participating in the pathogenesis of IgAN by inducing the release of inflammatory factors. On the other hand, it was also possible that our sample size was relatively small, and there might be new discoveries if we enlarged the sample size. Previous studies demonstrated that TNF- $\alpha$ was strongly correlated with mucosal immunity in IgAN [27-29]. TNF- $\alpha$ might be involved in podocyte changes and crescent formation and was related to the severity of IgAN renal interstitial fibrosis [30, 31]. TGF- $\beta 1$ had a dominant role in the accumulation of extracellular matrix and the development of renal tissue fibrosis in IgAN [32-35]. MCP-1 induced mesangial cell proliferation and had a crucial role in the progression of renal tubular injury and interstitial fibrosis [36-38], and its increased expression was associated with progressive tubulointerstitial inflammation $[39,40]$. Our results also suggested that TNF- $\alpha$, TGF- $\beta 1$, and MCP-1 have important roles in tubulointerstitial injury in IgAN, and this is consistent with previous research conclusions. Studies reported that TNF- $\alpha$ and TGF- $\beta$ were important regulators of IgAN mesangial-tubular crosstalk and mesangialpodocyte communication $[16,41,42]$. In this study, we investigated whether SIgA stimulated HRMC to secrete inflammatory cytokines that mediated IgAN tubulointerstitial damage through mesangial-tubular crosstalk. The specific regulatory factors involved and their mechanism of action remain to be further explored.

Taken together, our data suggested that the inflammatory mediators released from the glomerular mesangium induced by SIgA might be part of the mechanisms maintaining mesangial-tubular crosstalk during the development of tubulointerstitial damage in IgAN.

\section{Acknowledgements}

The authors sincerely thank Professor Zhang Hong and Professor Zhao Minghui of Peking University First Hospital for providing the experimental platform and technical guidance. The technical assistance and expertise of Quan Songxia and Zhou Yali are kindly acknowledged. Furthermore, we sincerely thank the biobank of the First Affiliated Hospital of Zhengzhou University for providing us with samples and thank Edanz Group China (www. liwenbianji.cn/ac) for editing the English text of a draft of this manuscript.

\section{Statement of Ethics}

The research was carried out according to the World Medical Association Declaration of Helsinki and was approved by the Ethics Committee of the First Affiliated Hospital of Zhengzhou University No. 2020-KY-216. Written informed consent was obtained from all subjects.

\section{Conflict of Interest Statement}

The authors have no conflicts of interests.

\section{Funding Sources}

This work was supported by grants from the National Natural Science Foundation of China (No. 81570645), the Innovation Scientists and Technicians Troop Construction Projects of Henan 
Province (No. 2018JR0014), the Sponsored by Program for Science and Technology Innovation Talents in Universities of Henan Province (No. 18HASTIT043), the Major Project of Henan Medical Science and Technology Research Program (No. 201501018), the Science and Technology Huimin Project of Henan Province (No. 162207310001), and the Pathogenesis and Precision diagnosis and treatment of CKD (No. 182101510002).

\section{Author Contributions}

Junjun Zhang and Zhangsuo Liu involved in conceiving and designing the experiments, as well as providing study materials or patients, manuscript review; Ruwen Zhou and Yiming Mi carried out the experiments, analysed the data, and wrote the manuscript. Bo Huang, Ruxue Guo, Panfei Wang, Songxia Quan, and Yali Zhou collected samples and assembled data.

\section{References}

1 Li LS, Liu ZH. Epidemiologic data of renal diseases from a single unit in China: analysis based on 13,519 renal biopsies. Kidney Int. 2004 Sep;66(3):920-3.

2 Magistroni R, D’Agati VD, Appel GB, Kiryluk K. New developments in the genetics, pathogenesis, and therapy of IgA nephropathy. Kidney Int. 2015 Nov;88(5):974-89.

3 Wyatt RJ, Julian BA. Renal vascular lesions in IgA nephropathy. J Renal Inj Prev. 2013 Jun; 2(2):37-8.

4 Suzuki H, Kiryluk K, Novak J, Moldoveanu Z, Herr AB, Renfrow MB, et al. The pathophysiology of IgA nephropathy. J Am Soc Nephrol. 2011 Oct;22(10):1795-803.

5 Oortwijn BD, van der Boog PJ, Roos A, van der Geest RN, de Fijter JW, Daha MR, et al. A pathogenic role for secretory IgA in IgA nephropathy. Kidney Int. 2006 Apr;69(7):11318

6 Zhang JJ, Xu LX, Liu G, Zhao MH, Wang HY. The level of serum secretory IgA of patients with IgA nephropathy is elevated and associated with pathological phenotypes. Nephrol Dial Transplant. 2008 Jan;23(1):207-12.

7 Tan Y, Zhang JJ, Liu G, Zhang H, Zhao MH. The level of urinary secretory immunoglobu$\operatorname{lin} \mathrm{A}(\operatorname{sg} \mathrm{A})$ of patients with IgA nephropathy is elevated and associated with pathological phenotypes. Clin Exp Immunol. 2009 Apr; 156(1):111-6.

8 Liang Y, Zhang J, Zhou Y, Xing G, Zhao G, Liu Z. Proliferation and cytokine production of human mesangial cells stimulated by secretory IgA isolated from patients with IgA nephropathy. Cell Physiol Biochem. 2015 Jul; 36(5):1793-808.

9 Rychlik I, Andrassy K, Waldherr R, Zuna I, Tesar V, Jancová E, et al. Clinical features and natural history of IgA nephropathy. Ann Med Interne. 1999 Feb;150(2):117-26.

10 D'Amico G, Imbasciati E, Barbiano Di Belgioioso G, Bertoli S, Fogazzi G, Ferrario F, et al. Idiopathic IgA mesangial nephropathy. Clinical and histological study of 374 patients. Medicine. 1985 Jan;64(1):49-60.

11 Zeng CH, Le W, Ni Z, Zhang M, Miao L, Luo $\mathrm{P}$, et al. A multicenter application and evaluation of the oxford classification of IgA nephropathy in adult chinese patients. Am J Kidney Dis. 2012 Nov;60(5):812-20.

12 Alexopoulos E, Seron D, Hartley RB, Nolasco F, Cameron JS. The role of interstitial infiltrates in IgA nephropathy: a study with monoclonal antibodies. Nephrol Dial Transplant. 1989;4(3):187-95.

13 Falk MC, Ng G, Zhang GY, Fanning GC, Roy LP, Bannister KM, et al. Infiltration of the kidney by alpha beta and gamma delta $\mathrm{T}$ cells: effect on progression in IgA nephropathy. Kidney Int. 1995 Jan;47(1):177-85.

14 Lv J, Shi S, Xu D, Zhang H, Troyanov S, Cattran DC, et al. Evaluation of the oxford classification of IgA nephropathy: a systematic review and meta-analysis. Am J Kidney Dis. 2013 Nov;62(5):891-9.

15 Shima Y, Nakanishi K, Hama T, Mukaiyama $\mathrm{H}$, Togawa $\mathrm{H}$, Hashimura $\mathrm{Y}$, et al. Validity of the oxford classification of IgA nephropathy in children. Pediatr Nephrol. 2012 May;27(5): 783-92.

16 Chan LY, Leung JC, Tsang AW, Tang SC, Lai $\mathrm{KN}$. Activation of tubular epithelial cells by mesangial-derived TNF-alpha: glomerulotubular communication in IgA nephropathy. Kidney Int. 2005 Feb;67(2):602-12.

17 Leung JC, Chan LY, Tang SC, Lam MF, Chow $\mathrm{CW}$, Lim AI, et al. Oxidative damages in tubular epithelial cells in IgA nephropathy: role of crosstalk between angiotensin II and aldosterone. J Transl Med. 2011 Oct;9:169.

18 Sun S, Zhao A, Li R, Du R, He L, Sun W, et al. CD147 renal expression as a biomarker for progressive IgAN. J Nephrol. 2015 Jun;28(3): $307-14$.

19 Xianyuan L, Wei Z, Yaqian D, Dan Z, Xueli T, Zhanglu D, et al. Anti-renal fibrosis effect of asperulosidic acid via TGF- $\beta 1 / \mathrm{smad} 2 / \mathrm{smad} 3$ and NF- $\mathrm{kB}$ signaling pathways in a rat model of unilateral ureteral obstruction. Phytomedicine. 2019 Feb;53:274-85.

20 Xiao J, Leung JC, Chan LY, Tang SC, Lai KN. Crosstalk between peroxisome proliferatoractivated receptor-gamma and angiotensin II in renal tubular epithelial cells in IgA nephropathy. Clin Immunol. 2009 Aug;132(2): 266-76.

21 Xiao J, Leung JC, Chan LY, Guo H, Lai KN. Protective effect of peroxisome proliferatoractivated receptor-gamma agonists on activated renal proximal tubular epithelial cells in IgA nephropathy. Nephrol Dial Transplant. 2009 Jul;24(7):2067-77.

22 Lai KN, Chan LY, Leung JC. Mechanisms of tubulointerstitial injury in IgA nephropathy. Kidney Int Suppl. 2005 Apr;(94):S110-5.

23 Kerr MA. The structure and function of human IgA. Biochem J. 1990 Oct;271(2):285-96.
24 Mathias A, Corthésy B. Recognition of gram-positive intestinal bacteria by hybridoma- and colostrum-derived secretory immunoglobulin A is mediated by carbohydrates. J Biol Chem. 2011 May;286(19): 17239-47.

25 Liang Y, Zhao G, Tang L, Zhang J, Li T, Liu Z. MiR-100-3p and miR-877-3p regulate overproduction of IL- 8 and IL- $1 \beta$ in mesangial cells activated by secretory IgA from IgA nephropathy patients. Exp Cell Res. 2016 Oct; 347(2):312-21.

26 Moura IC, Arcos-Fajardo M, Sadaka C, Leroy V, Benhamou M, Novak J, et al. Glycosylation and size of IgA1 are essential for interaction with mesangial transferrin receptor in IgA nephropathy. J Am Soc Nephrol. 2004 Mar; 15(3):622-34.

27 Kiryluk K, Li Y, Scolari F, Sanna-Cherchi S, Choi M, Verbitsky M, et al. Discovery of new risk loci for IgA nephropathy implicates genes involved in immunity against intestinal pathogens. Nat Genet. 2014 Nov;46(11): 1187-96.

$28 \mathrm{He} \mathrm{B}, \mathrm{Xu} \mathrm{W}$, Santini PA, Polydorides AD, Chiu A, Estrella J, et al. Intestinal bacteria trigger T cell-independent immunoglobulin A(2) class switching by inducing epithelial-cell secretion of the cytokine APRIL. Immunity. 2007 Jun;26(6):812-26.

29 Kiryluk K, Novak J. The genetics and immunobiology of IgA nephropathy. J Clin Invest. 2014 Jun;124(6):2325-32.

30 Sasaki Y, Shimizu Y, Suzuki Y, Horikoshi S, Tomino Y. TWEAK/Fn14 system and crescent formation in IgA nephropathy. BMC Nephrol. 2015 Mar;16:27.

31 Sonoda Y, Gohda T, Suzuki Y, Omote K, Ishizaka M, Matsuoka J, et al. Circulating TNF receptors 1 and 2 are associated with the severity of renal interstitial fibrosis in IgA nephropathy. PLoS One. 2015 Apr; 10(4): e0122212.

32 Fukasawa H, Yamamoto T, Suzuki H, Togawa A, Ohashi N, Fujigaki Y, et al. Treatment with anti-TGF-beta antibody ameliorates chronic progressive nephritis by inhibiting Smad/ TGF-beta signaling. Kidney Int. 2004 Jan; 65(1):63-74.

33 Yamamoto T, Noble NA, Cohen AH, Nast CC, Hishida A, Gold LI, et al. Expression of transforming growth factor-beta isoforms in human glomerular diseases. Kidney Int. 1996 Feb;49(2):461-9. 
34 Wada J, Sugiyama H, Makino H. Pathogenesis of IgA nephropathy. Semin Nephrol. 2003 Nov;23(6):556-63.

35 Wynn TA. Cellular and molecular mechanisms of fibrosis. J Pathol. 2008 Jan;214(2): 199-210.

36 Tesch GH, Schwarting A, Kinoshita K, Lan HY, Rollins BJ, Kelley VR. Monocyte chemoattractant protein-1 promotes macrophage-mediated tubular injury, but not glomerular injury, in nephrotoxic serum nephritis. J Clin Invest. 1999 Jan;103(1):73-80.
37 Sun Y, Yuan S, Xu X. [Expression of MCP-1 in renal tissues of patients with IgA nephropathy]. Zhong Nan Da Xue Xue Bao Yi Xue Ban. 2009 Oct;34(10):1023-8.

38 Kuper C, Beck FX, Neuhofer W. Autocrine MCP-1/CCR2 signaling stimulates proliferation and migration of renal carcinoma cells. Oncol Lett. 2016 Sep;12(3):2201-9.

39 Segerer S, Alpers CE. Chemokines and chemokine receptors in renal pathology. Curr Opin Nephrol Hypertens. 2003 May;12(3): 243-9.

40 Tesch GH. MCP-1/CCL2: a new diagnostic marker and therapeutic target for progressive renal injury in diabetic nephropathy. Am J Physiol Renal Physiol. 2008 Apr;294(4): F697-701.
41 Lai KN, Leung JC, Chan LY, Saleem MA, Mathieson PW, Lai FM, et al. Activation of podocytes by mesangial-derived TNF-alpha: glomerulo-podocytic communication in IgA nephropathy. Am J Physiol Renal Physiol. 2008 Apr;294(4):F945-55.

42 Lai KN, Leung JC, Chan LY, Saleem MA, Mathieson PW, Tam KY, et al. Podocyte injury induced by mesangial-derived cytokines in IgA nephropathy. Nephrol Dial Transplant. 2009 Jan;24(1):62-72. 